\title{
¿Estrellas o constelaciones? Implicaciones de los estudios cognitivos para el modelo dimensional de la emoción
}

\section{Stars or constellations? Implications of cognitive studies for the dimensional model of emotion}

\author{
Antonio Olivera-La Rosa ${ }^{1,2}$, Olber Eduardo Arango Tobón ${ }^{1}$ y Juan José Martí Noguera ${ }^{3}$ \\ ${ }^{1}$ Departamento de Psicología y Ciencias Sociales, Fundación Universitaria Luis Amigó, Colombia. \\ ${ }^{2}$ Human Evolution and Cognition Group, grupo asociado a IFISC, Universidad de las Islas Baleares - CSIC, España. \\ ${ }^{3}$ Departmento de Psicología Básica, Universidad de Valencia, España.
}

Disponible online 31 de agosto de 2016

\begin{abstract}
Uno de los principales debates dentro del estudio de la psicología de la emoción concierne a la concepción de las emociones como constructos psicológicos unificados (categoriales/discretos) en contraposición con el enfoque dimensional del episodio emocional. En este marco, el modelo dimensional de Russell (2003) destaca en el panorama académico al constituir una propuesta integradora que da cuenta de una serie de problemas históricos del ámbito de estudio. Con este fin, se realiza una aproximación analítica a dicho modelo y se argumenta su viabilidad. El presente artículo tiene como objetivo revisar la evidencia empírica a favor de cuatro hipótesis que sostiene el modelo dimensional de Russell: (1) Existe una primacía temporal de los afectos básicos frente a los componentes más cognitivos en el desarrollo de la emoción, (2) los cambios afectivos percibidos facilitan la accesibilidad del material hedónicamente congruente, (3) la mente humana puede atribuir erróneamente la experimentación de un determinado core affect al estímulo incorrecto, y (4) la experimentación de los componentes dimensionales facilita la ocurrencia de respuestas cognitivas congruentes. Se concluye que la combinación de componentes dimensionales inespecíficos, tanto de tipo puramente afectivo como de carácter más "cognitivo" constituyen ocasionalmente los prototipos emocionales. En consecuencia, se propone que el modelo dimensional de Russell (2003) constituye una alternativa integradora de gran potencial para abordar el estudio de la naturaleza de la emoción.
\end{abstract}

Palabras Clave: Emoción; Cognición; Core Affect; Modelo Dimensional.

One of the main debates in the study of the psychology of emotion concerns the conception of emotions as unified psychological constructs (categorical/discrete) versus the dimensional approach to emotional episodes. In this context, the dimensional model of Russell (2003) stands out as an inclusive framework that takes into account a set of historical problems in this field of study. We analysed the model, discussed its viability, and reviewed the empirical evidence in favour of four hypotheses that underlie Russell's dimensional model: (1) There is a temporary primacy of basic affects versus more cognitive components in the development of an emotion; (2) perceived affective changes facilitate access to hedonically congruent material; (3) the human mind can mistakenly attribute the experience of a specific core affect to the incorrect stimulus; and (4) the experience of the dimensional components facilitates the occurrence of congruent cognitive responses. The combination of nonspecific dimensional components, both purely affective as well as those with more "cognitive" characteristics, can act as prototypical emotions. We conclude that Russell's dimensional model is an integrated alternative to other models with great potential for studying the nature of emotion.

Key Words: Emotion; Cognition; Core Affect; Dimensional Model

Correspondencia: Antonio Olivera-La Rosa. Fundación Universitaria Luis Amigó- Calle 74 No. 97-95 Zona Sur - Barrio LA Navarra. Urabá-Colombia. Email: antonio.oliverade@amigo.edu.co / acensulay@yahoo.es; . E-mails de los co-autores: Olber Eduardo Arango Tobón: eduarango@une.net. co.; Juan José Martí Noguera: juanjosemarti@uan.edu.co. 
¿Qué es exactamente una emoción? Desde que William James (1884) se formulara esta pregunta, pasando por las tesis seminales de Darwin (1872), comprender la naturaleza de lo que se entiende académicamente como "emoción" ha presentado un auténtico reto para la psicología. Y es que, a diferencia de otros ámbitos de investigación, en el estudio de la emoción parece existir un patrón periódico de cuestionamiento de la validez científica de ciertos conceptos emocionales. Este hecho no es gratuito, pues el estudio de la emoción ha arrastrado históricamente una problemática conceptual, y en menor medida, metodológica, que ha sumido a este ámbito de estudio en un estado constante de polémica (Lindquist, Wager, Kober, BlissMoreau, y Barrett, 2012; Rosselló y Revert, 2008).

Ciertamente, si algo parece evidente es que las emociones desempeñan un rol fundamental en las experiencias humanas. Como señala Dolan (2002), las emociones desempeñan un papel crucial en la dinámica de las relaciones sociales, constituyendo la principal fuerza motivacional que subyace a una serie de evaluaciones y conductas. En este particular contexto, uno de los ámbitos que ha despertado mayor interés en la comunidad académica es el estudio de la interacción entre los procesos emocionales y otros dominios de la cognición. En efecto, parece ser que la naturaleza de las emociones resulta de alguna manera menos "encapsulada" que la de otras respuestas psicológicas, hecho que se manifiesta en los efectos globales de las mismas en virtualmente todos los aspectos cognitivos (para revisiones, ver Meier, Schnall, Schwarz y Bargh, 2012; Schwarz, 2011). A nivel neural, la evidencia actual sugiere que los procesos emocionales y cognitivos no son procesos claramente separables, por el contrario, la constante interacción entre las bases neurales de ambos procesos sugiere que estas deben ser entendidas como fuertemente no-modulares ( $\mathrm{Gu}$, Liu,Van Dam, Hof, y Fan, 2013; Phelps, 2006).

El objetivo del presente trabajo consiste en revisar la evidencia empírica que sustenta al modelo dimensional de Russell (2003). Bajo esta premisa, el artículo está planteado como una revisión crítica (no sistemática) de la literatura y, en consecuencia, no se tomarán en cuenta aquellos estudios que no constituyan fundamento empírico para las hipótesis derivadas del modelo de Russell (para revisar estudios meta-analíticos sobre el debate discreto-dimensional ver, Lindquist, Wager, Kober, Bliss-Moreau, y Barrett, 2012; Vital y Hamann, 2010).

La estructura del artículo se divide en tres partes. El primer apartado resume a modo de marco contextual los postulados teóricos de los dos principales enfoques existentes sobre la naturaleza psicológica de la emoción: el enfoque discreto y el enfoque dimensional. Posteriormente, se examina la evidencia empírica - proveniente de la investigación cognitiva- que concierne a cuatro hipótesis derivadas del enfoque dimensional de Russell (2003). Para finalizar, se discuten las implicaciones teóricas de los estudios analizados y se propone un modelo integrador de la naturaleza de la emoción.

\section{Modelos teóricos de la naturaleza de la emoción}

En este contexto, un tema central de la problemática concierne a la naturaleza de lo que es en términos psicológicos una emoción. Por ejemplo, ¿es el miedo un constructo psicológico unificado o por el contrario debe ser entendido como la experiencia resultante de la integración de diferentes componentes cognitivos/afectivos? Si bien el debate es ciertamente complejo y contempla una serie de posiciones intermedias (Barrett, 2012), los diferentes postulados se pueden dividir en dos grandes marcos conceptuales. Por un lado, existe el marco teórico que entiende a las emociones como categorías discretas que poseen un estatus "básico", el cual se manifiesta de forma diferencial tanto a un nivel expresivo, como cognitivo y neural (Ekman y Cardoro, 2011; Izard, 2011). En contraposición a este enfoque, diversos autores consideran que la mejor manera de entender las emociones es como el resultado emergente de la combinación de diferentes componentes dimensionales (Russell, 2003).

\section{Enfoque discreto (o categorial) de la emoción}

El primer modelo asume que existen emociones básicas (alegría, tristeza, miedo, sorpresa, ira y repugnancia; entre las ampliamente aceptadas, Rosselló y Revert, 2008) de carácter universal, cuyas expresiones faciales son representadas por los mismos músculos faciales a nivel transcultural (Ekman, 1999). A nivel cognitivo, estas emociones se caracterizan por patrones específicos de valoración (appraisal) que implican el "significado" del evento emocional; en otras palabras, la ocurrencia de una determinada emoción dependerá de la interpretación que se le dé a la situación (por ejemplo, si se percibe una situación como peligrosa se experimentará miedo; Ellsworth y Scherer, 2003). Adicionalmente, los partidarios del enfoque discreto de la emoción defienden la existencia de sistemas neurales involucrados de forma diferenciada en cada proceso emocional, en otras palabras, se asume que cada emoción está representada de forma específica a nivel neural (Calder, Lawrence y Young, 2001).

El enfoque discreto de las emociones ha tenido una aceptación significativa en la comunidad académica, no obstante, existen estudios que ponen en entredicho algunos de sus postulados (para una revisión, ver Barrett, 2012). Por ejemplo, no existe una correspondencia unívoca entre emociones básicas y conductas específicas, inclusive entre animales no-humanos (Lin et al., 2011; Motta et al., 2009). Ciertamente, los estudios en estimulación eléctrica de neuronas han encontrado que los efectos de estas manipulaciones no producen respuestas emocionales específicas (Kringelbach, Green, Owen, Schweder y Aziz, 2010). En esta línea, el estudio de las lesiones cerebrales ha evidenciado que las personas que sufren lesiones en áreas cerebrales no suelen presentar déficits emocionales específicos (Beer, John, Scabini y Knight, 2006; Calder et al., 2010). Inclusive, la evidencia actual es contradictoria en lo que respecta a la correspondencia unívoca asumida tradicionalmente entre 
ciertas áreas cerebrales y ciertas emociones (Hamann, 2012; Scarantino, 2012). Por ejemplo, si bien la amígdala ha sido repetidamente asociada con la respuesta de miedo (Bach, Hurlemann, y Dolan, 2015), existen estudios que cuestionan seriamente la especificad de este postulado (Ousdal, Andreassen, Server y Jensen, 2014; Tsuchiya, Moradi, Felsen, Yamazaki y Adolphs, 2009). Adicionalmente, no existe evidencia de que la experiencia de una emoción conlleve cambios a nivel biológico que resulten consistentes y específicos para cada categoría emocional (Moons, Eisenberger y Taylor, 2010). Por último, el reconocimiento universal de las emociones discretas ha demostrado ser altamente susceptible a aspectos metodológicos. Ciertamente, la inclusión de conceptos (palabras) emocionales en los estudios de identificación emocional parece ser un factor diferencial cuando se trata de encontrar posibles interpretaciones universales de las expresiones faciales (Gendron, Roberson, van der Vyver, y Barrett, 2014; Widen, Christy, Hewett, y Russell, 2011).

\section{El enfoque dimensional de la emoción}

Existe un segundo enfoque teórico para abordar la naturaleza del episodio emocional: el enfoque dimensional. Esta perspectiva presupone la existencia de ciertas dimensiones inespecíficas o "primitivos emocionales" que subyacen a todo episodio emocional definiéndolo, a su vez, cualitativamente. Por consiguiente, las emociones no son concebidas como respuestas discretas diferenciables a nivel psicológico, sino como representaciones emergentes de la experimentación de componentes dimensionales inespecíficos (Lindquist et al., 2012; Russell y Barrett, 1999; Wilson-Mendenhall, Barrett y Barsalou, 2013).

Cabe destacar que ciertos autores han criticado los enfoques dimensionales al atribuirles una caracterización reduccionista de la emoción, pues ciertas propuestas parecen mitigar la importancia del componente cualitativo de la emoción en favor de sus componentes puramente afectivos/fisiológicos (Reisenzein, 1994). En particular, un aspecto importante de estas críticas se centra en la aparente incapacidad de los enfoques dimensionales para explicar las obvias diferencias entre las emociones discretas. Según los detractores de este modelo, las fluctuaciones en la dimensión de placer-displacer o de la activación experimentada (las cuales son asumidas ampliamente como las dimensiones básicas inherentes a toda emoción; Lang, 1980; Russell y Barrett, 1999) no pueden dar cuenta de las diferencias cualitativas existentes entre ciertas emociones (por ejemplo, tanto el miedo como la ira o la repugnancia son todos estados activantes de naturaleza afectiva negativa).

\section{El modelo de Russell: una propuesta integradora}

\section{Principales hipótesis del modelo de Russell}

En esta línea, la propuesta del core affect (Russell, 2003) destaca por su capacidad para solventar algunas de las históricas controversias en el debate discreto-dimensional. Así, la propuesta de Russell se distingue entre las perspectivas dimensionales al considerar que el core affect (entendido como un estado afectivo inespecífico) constituye una parte necesaria más no suficiente del episodio emocional.

En particular, el core affect debe ser entendido como un estado neurofisiológico de tipo básico e inespecífico, subyacente a toda experiencia emocional. Es importante señalar que si bien el core affect es experimentado conscientemente como un sentimiento integrado, en realidad deriva de la combinación de valores hedónicos (placer-displacer) y de arousal (activación-relajación). En consecuencia, el concepto de core affect se encuentra estrechamente relacionado con los conceptos de sentimiento subjetivo ("feeling") y de humor ("mood"), entendido este último como el tono general ("free-floating") de la respuesta afectiva a lo largo del tiempo (Clark \& Isen, 1982). La analogía utilizada por Russell (2009) con la sensación de percibir un color puede ser útil para comprender mejor el concepto: las dimensiones de tono, saturación y brillo se combinan de forma integral para formar la sensación unificada de un color específico.

Por consiguiente, la diferenciación entre emociones discretas (o prototipos emocionales) radicará en la interacción del core affect con otros componentes dimensionales: atribución, cualidad afectiva, appraisal, tendencia a la acción, manifestación expresiva/fisiológica y el componente de experiencia subjetiva.

El componente de atribución es entendido como el proceso mediante el cual la experimentación de un determinado core affect es percibido como la respuesta ante un estímulo-objeto, el cual implícitamente se asume que posee una cualidad (u potencial) afectiva. El segundo componente (appraisal) consiste en la valoración subjetiva que se realiza del mencionado objeto. La tendencia a la acción es entendida como la predisposición conductual que resulta de la valoración del objeto. La manifestación expresiva/fisiológica da cuenta de los cambios somáticos, faciales y vocales. Finalmente, la dimensión de la experiencia subjetiva (meta-experiencia emocional) se refiere a la categorización que se le otorga al estado emocional, es decir, la experiencia fenomenológica que resulta de la integración de los componentes mencionados.

Ciertamente, la perspectiva de Russell se nutre en parte del modelo "periférico" de James (1884) que entendía a la emoción como el resultado de la auto-percepción de procesos automáticos de tipo fisiológico. De forma más directa, la propuesta de Russell también se identifica con la teoría bifactorial de Schachter y Singer (1962) según la cual la emoción resulta de una activación fisiológica inespecífica y de la subsiguiente valoración cognitiva de la situación en que ocurre dicha activación. Asimismo, el concepto de core affect es teóricamente compatible con las teorías de la cognición corporizada (embodiment), las cuales sostienen que los procesos cognitivos complejos se nutren de la información proveniente de nuestro cuerpo (Damasio, 1999; Meier et al., 2012).

En esta línea, Russell (2003) considera que si bien este 
primer primitivo emocional (core affect) es per se inespecífico -Russell lo compara con la temperatura corporal-, puede ocasionalmente ser atribuido a alguna causa y desencadenar así el principio del episodio emocional. El segundo primitivo emocional consiste en la percepción de la cualidad afectiva (affective quality), la cual es entendida como una cualidad inherente al estímulo con el potencial para influir en el core affect. De esta forma, mientras que el core affect existe a nivel interno ("tengo una sensación inquietante") la cualidad afectiva existe en el estímulo ("es el león lo que me inquieta").

Desde esta perspectiva, a partir de la experimentación del core affect $\mathrm{y}$ de la percepción de la cualidad afectiva se puede definir todo lo referente a la vida emocional. En concreto, para Russell (2003) la vida emocional consiste en las fluctuaciones constantes del core affect como resultado de la percepción igualmente constante de cualidades afectivas, así como en la frecuente atribución del core affect a un único objeto, fenómenos que en interacción con otros componentes perceptuales, cognitivos y conductuales forman ocasionalmente alguno de los patrones prototípicos emocionales. En consecuencia, las emociones "discretas" no existen como tal a nivel psicológico, pues en realidad son el resultado de la coincidencia entre una serie de componentes (core affect, percepción de cualidades afectivas, atribución del core affect a un objeto, etc.) y una representación mental (el "prototipo" emocional). Las emociones, por tanto, deben ser entendidas como patrones prototípicos emocionales derivados de los componentes dimensionales, de la misma forma que las constelaciones son el resultado emergente de las estrellas (Russell, 2003, p. 152).

\section{Evidencia empírica del modelo}

Como se mencionó anteriormente, la perspectiva dimensional de Russell (2003) solventa parte de las controversias imputadas tradicionalmente a los enfoques dimensionales; en particular, aquellas que atribuían a estas propuestas una concepción reduccionista de la emoción. No obstante, se considera necesario contrastar ciertos postulados teóricos de este modelo incorporando los estudios más recientes en cognición y emoción.

En este contexto, si se asume que el modelo dimensional de Russell da buena cuenta de la entidad psicológica de la emoción, es posible derivar una serie de hipótesis específicas relacionadas a esta concepción dimensional del episodio emocional. Primero, si los cambios en el core affect basal constituyen la primera instancia de todo episodio emocional, debería existir una primacía temporal de los afectos básicos frente a los componentes más cognitivos en el desarrollo de la emoción (Hipótesis 1). Segundo, si los cambios percibidos en el core affect generan automáticamente una búsqueda de los estímulos causantes, es esperable que este mecanismo facilite a su vez la accesibilidad del material hedónicamente congruente (Hipótesis 2). Tercero, y en relación con el punto anterior, si la experimentación del core affect es un proceso distinguible de la percepción de la cualidad afectiva (proceso “cold"), es factible que ambos procesos resulten disociados en ciertas situaciones en las cuales la mente atribuye erróneamente la experimentación de un determinado core affect al estímulo incorrecto (Hipótesis 3). Finalmente, si los diferentes componentes dimensionales involucrados en el episodio emocional son inespecíficos, se podría esperar que el experimentar estas dimensiones facilite respuestas cognitivas congruentes, independientemente del core affect (Hipótesis 4).

Hipótesis 1: Primacía de los afectos básicos en el desarrollo temporal del episodio emocional. Un postulado central de la teoría dimensional de Russell es que la auto-percepción de los cambios en el core affect supone la primera instancia de todo episodio emocional. Por consiguiente, a efectos del desarrollo temporal (time-course) de la emoción, este postulado implica necesariamente que los componentes afectivos de tipo más básico (ej.: valencia/activación) preceden a aquellos componentes dimensionales de tipo más "cognitivo" (ej.: atribución, appraisal). Ciertamente, esta postura se encuentra en línea con los modelos teóricos que defienden la primacía del afecto (Zajonc, 1980; 2000) y la creencia de que la evaluación automática de los estímulos antecede a las valoraciones más específicas en el procesamiento de la información afectiva. Dicho de otro modo, cuando percibimos un estímulo, el procesamiento de la información típicamente va de lo global-evaluativo a lo local-descriptivo (Almeida, Pajtas, Mahon, Nakayama \& Caramazza, 2013).

Los resultados obtenidos a partir de la investigación en priming afectivo han resultado especialmente útiles en este tema. En efecto, el estado de la cuestión favorece que la utilización de tiempos de exposición extremadamente breves dan lugar a respuestas afectivas básicas; conversamente, las respuestas cognitivas de tipo más complejo requieren de más tiempo para ser activadas (Almeida et al., 2013; Murphy y Zajonc, 1993; Wong y Root, 2003).

Complementariamente, diversos estudios sugieren que la dimensión de activación (arousal) es de especial relevancia -nunca mejor dicho- en las primeras instancias de procesamiento emocional. Así, parece ser que la respuesta de arousal es generada de forma involuntaria y no requiere de mayor tiempo para ser activada (Gibbons, 2009; Kuchinke, Krause, Fritsch y Briesemeister, 2014; Rohr, Degner y Wentura, 2014). Por ejemplo, Schimmack y Derryberry (2005) encontraron que el grado de activación fue el principal predictor del nivel de interferencia atencional derivado de una serie de imágenes emocionales. Según el autor, estos resultados sugieren que el primer appraisal de un estímulo afectivo estaría relacionado con la relevancia y el arousal (para una conclusión similar, ver Scherer, 2001).

A nivel neurológico, diversos estudios han investigado los correlatos neurales del curso temporal del procesamiento de las expresiones faciales. Paulmann y Pell (2009) encontraron que la información puramente afectiva es accesible al procesamiento neocortical dentro de los $200 \mathrm{~ms}$ que preceden a la 
percepción del estímulo emocional. En este estudio, se documentó que la valoración semántica del mismo estímulo (una expresión facial) no fue procesada hasta aproximadamente los $400 \mathrm{~ms}$. Asimismo, se ha documentado que existen sistemas independientes a nivel neural para el procesamiento puramente evaluativo y para el procesamiento de tipo más detallado (Adolphs, 2003; ver también Wilson-Mendenhall et al., 2013). En la misma línea, existe evidencia de que la percepción de expresiones faciales genera rápidamente categorizaciones afectivas, mientras que los procesos implicados en el reconocimiento de la identidad (y el contexto) de la expresión requiere de un mayor tiempo (Atkinson y Adolphs, 2005; Diéguez-Risco, Aguado, Albert \& Hinojosa, 2013; Palermo y Rhodes, 2007).

En conjunto, tanto los estudios cognitivos como los desarrollados a nivel neuropsicológico sugieren que la información emocional es procesada en una primera instancia a nivel globalevaluativo (core affect) para progresivamente incorporar a las propiedades más específicas y descriptivas del estímulo. Esta conclusión es consecuente con la primacía del core affect como fundamento básico de todo episodio emocional.

Hipótesis 2: Los cambios en el core affect facilitan la accesibilidad del material hedónicamente congruente. El segundo postulado a examinar de la teoría de Russell sostiene que los cambios en el core affect -especialmente cuando estos son repentinos o drásticos- son objeto inmediato de los recursos atencionales, respuesta que se sustenta en la necesidad de encontrar rápidamente posibles causas de la alteración del afecto basal. Como consecuencia derivada, este mecanismo facilita que el procesamiento del material hedónicamente congruente resulte más accesible. Este postulado está en línea con el modelo clásico de red semántica de la emoción de Bower (1981), según el cual los estados de ánimo facilitan la activación y la accesibilidad de las cogniciones asociadas con tales estados de ánimo. Por ejemplo, está ampliamente documentado que la propensión a experimentar ansiedad está asociada con la tendencia a incrementar la atención hacia los estímulos de naturaleza aversiva (Hermans, Spruyt, De Houwer y Eelen, 2003).

El estudio de esta particularidad se ha visto reforzado por el especial interés que ha existido en la última década para estudiar los mecanismos subyacentes al efecto del priming afectivo. En este contexto, uno de los principales candidatos a mecanismo operativo en este efecto es conocido como spreading activation, el cual asume que el priming "activa" un determinado constructo mental (cargado hedónicamente) que se encuentra interconectado - a manera de nodos mnemónicoscon otros constructos mentales con los cuales comparte alguna propiedad en común (semántica y/o afectiva). De esta manera, son diversos los estudios que han documentado de que el hecho de experimentar un determinado estado afectivo genera que se categorice de forma más rápida aquellos estímulos que son congruentes con dicho estado (Niedenthal, 2008; Wong y Root, 2003). Por mencionar algunos ejemplos, Lodge y Taber (2005) encontraron que los tiempos de reacción fueron menores para targets (conceptos políticos) que resultaban afectivamente congruentes con los primes (pese a no tener relación semántica alguna; ver también, Rohr, Degner, y Wentura, 2012). Adicionalmente, se han encontrado efectos de congruencia entre el estado de ánimo inducido ("tristeza") y el procesamiento de palabras de dicha categoría (Meilán et al., 2012) y, de forma similar, se ha documentado de que la percepción de expresiones faciales de miedo disminuyó los tiempos de reacción para identificar expresiones similares afectivamente (Yang, Xu, Du, Shi y Fang, 2011). Por consiguiente, se considera que la ubiquidad del "efecto priming" (reducción de los tiempos de reacción ante estímulos hedónicamente congruentes) en la literatura supone un soporte consistente para la segunda hipótesis.

Hipótesis 3: Ocasionalmente la mente puede atribuir erróneamente la experimentación de un determinado core affect a la percepción del estímulo incorrecto. En la propuesta dimensional de Russell (2003), un aspecto central es la relación existente entre los dos primitivos emocionales: la experimentación del core affect y la percepción de la cualidad afectiva. En este sentido, si bien ambos procesos se encuentran estrechamente ligados, Russell enfatiza que se tratan de dos procesos independientes dado que, mientras que el core affect se experimenta, la percepción de la cualidad afectiva implica en estricto un acto de representación.

Un fenómeno ampliamente documentado es que la mente humana típicamente atribuye que las respuestas cognitivas experimentadas son "acerca de" los estímulos que son percibidos en contigüidad temporal (Higgins, 1998). Así, sucede que ocasionalmente ciertas experiencias perceptuales son desligadas de su contexto original y terminan ejerciendo una influencia tanto en el procesamiento de la información subsecuente como en las respuestas conductuales derivadas. Esta particularidad ha sido abordada como parte del fenómeno de la "mala atribución" cognitiva (misattribution), según el cual nuestra mente atribuye las respuestas cognitivas a aquellos estímulos que son percibidos conscientemente en lugar de a aquellos que resultan inaccesibles a la consciencia (Paéz y Carbonero, 1993).

Los errores de atribución de las experiencias afectivas a las evaluaciones de preferencia ha sido, y continua siendo, el objeto de estudio de diferentes marcos conceptuales dentro de la psicología cognitiva (Doyen, Klein, Simons y Cleeremans, 2014). En la última década, la investigación en cognición social implícita ha revelado la importancia del "Affective Misattribution Procedure" (Payne, Cheng, Govorum y Stewart, 2005) como paradigma experimental. Este procedimiento es una variante del paradigma de Murphy y Zajonc (1993) el cual consiste en presentar a los participantes estímulos afectivamente neutros (ideogramas chinos) precedidos por estímulos primes de valencia afectiva positiva o negativa. El efecto típico es encontrar que los participantes prefirieren y juzgan como más positivos aquellos ideogramas precedidos por primes de valencia posi- 
tiva, mientras que los primes de valencia negativa disminuyen la valoración de los ideogramas (Blaison, Imhoff, Hühnel, Hess y Banse, 2012; Payne et al., 2005; ver también Gawronski y Ye, 2014).

Por ejemplo, se ha reportado que los primes afectivos de expresiones faciales (de felicidad y repugnancia) influenciaron las evaluaciones de obras artísticas de naturaleza abstracta de forma positiva y negativa, respectivamente (Flexas, Rosselló, Christensen, Nadal, Olivera La Rosa y Munar, 2013). En la misma línea, existe evidencia de que las expresiones faciales emocionales procesadas de forma subliminal afectan las evaluaciones de las expresiones neutras (Prochnow et al., 2013). Inclusive, Winkielman, Berridge y Wilbarger (2005) encontraron que la presentación subliminal de imágenes de sonrisas incrementó la predisposición de los participantes a servir, consumir, e incluso pagar más dinero por un brebaje. Interesantemente, los autores también encontraron que la presentación subliminal de ceños fruncidos produjo el efecto contrario en los participantes.

Dentro del marco de la cognición moral, son diversos los estudios que han encontrado un efecto de los afectos incidentales (aquellos que no son elicitados por las propiedades del estímulo emocional) en los juicios morales. Por ejemplo, la investigación en los efectos de los estímulos estéticos en la cognición moral ha encontrado que la percepción de rostros atractivos incrementó la honestidad de los participantes (Wang et al., 2015), un resultado congruente con la existencia implícita (pero ampliamente operativa) del estereotipo "si es bello es bueno" (Shannon y Shark, 2003).

En esta línea, se encontró que aquellos jurados que fueron inducidos a experimentar ira realizaron atribuciones más severas que aquellos jurados que se encontraban en un estado afectivo relativamente neutral (Lerner, Goldberg y Tetlock, 1998) Asimismo, se obtuvo evidencia de que el hecho de percibir brevemente imágenes negativamente impactantes redujo exclusivamente la severidad de los juicios morales (Olivera La Rosa y Rosselló, 2012) y de que la experiencia gustativa de repugnancia incrementa la severidad de los mismos (Eskine, Kacinik y Prinz, 2011). Curiosamente, parece ser que el efecto opuesto también resulta operativo en la cognición moral (Lee y Schwarz, 2010; Schnall, 2011; Schnall, Benton y Harvey, 2008).

Finalmente, los estudios realizados en el marco de los correlatos psicológicos (afectivos) de la experiencia musical sugieren que existe una estrecha conexión entre la música y la moralidad. Así, se ha documentado que la música tiene un efecto en la conducta moral, favoreciendo las respuestas empáticas, la pro-sociabilidad y el altruismo (Fukui y Toyoshima, 2014; Kirschner y Tomasello, 2010; Kokal, Engel, Kirschner y Keysers, 2011).Más aun, existe evidencia de que diferentes respuestas afectivas inducidas a través de composiciones musicales influenciaron de forma congruente a los juicios morales (Seidel y Prinz, 2013a, 2013b).
Hipótesis 4: los componentes dimensionales involucrados en el episodio emocional pueden influir congruentemente las respuestas cognitivas. Como se mencionó en el primer apartado, la aproximación dimensional de Russell (2003) se distingue de otras perspectivas dimensionales por incorporar en su modelo de la emoción a componentes dimensionales de tipo más "cognitivo". Así, tenemos que cada emoción prototípica involucra una determinada combinación de componentes dimensionales, algunos de tipo más "básico" (como el core affect) y otros de tipo más cognitivo (como el appraisal). En nuestra vida emocional cotidiana, ambos tipos de componentes se encuentran en estrecha relación; es decir, tanto el core affect influye a los appraisals subsecuentes como viceversa (Kuppens, Champagne, y Tuerlinckx, 2012).

Por ejemplo, pese a que el miedo y la ira son dos constructos emocionales que involucran un core affect similar (valor hedónico negativo y alta activación), su diferencia radica en otros componentes emocionales como el appraisal o la tendencia a la acción. Este hecho se ha documentado empíricamente en los estudios realizados a partir del "Appraisal-tendency framework" (Han, Lerner, y Keltner, 2007; Lerner y Keltner, 2001). Dentro de este marco teórico, los patrones específicos que constituyen el appraisal de cada emoción tienen la capacidad de influenciar los juicios evaluativos de forma congruente con las características de los patrones cognitivos involucrados. Retomando la comparación entre la emoción prototípica de miedo e ira, mientras que la primera involucra appraisals de incertidumbre, la segunda implica appraisals de control individual de la situación (Smith y Ellsworth, 1985). Consecuentemente, Lerner y Keltner (2001) encontraron que los participantes predispuestos a experimentar miedo presentaron una tendencia a realizar evaluaciones pesimistas, mientras que la experiencia de ira favoreció que los participantes se decantasen por evaluaciones de sesgo optimista.

En esta línea, un componente central del appraisal de la ira es la atribución de agencia. En efecto, Yang y Tong (2010) encontraron que aquellos participantes que fueron expuestos a una inducción de ira evidenciaron mayor tendencia a evaluar una serie de eventos negativos como causados por otras personas, mientras que la inducción de tristeza generó que los mismos eventos sean atribuidos a factores situacionales (para un resultado similar, ver también Keltner, Ellsworth y Edwards, 1993). Desde la misma perspectiva, Oveis, Horberg y Keltner (2010) encontraron que mientras que la experiencia de compasión favorece la sensación de similitud con aquellas personas que percibimos como "débiles", la experiencia de orgullo genera el mismo efecto empático frente a quienes identificamos como "fuertes". Además, parece ser que la experiencia emocional de "elevación" (la cual es descrita como una emoción positiva generada por la percepción de un acto virtuoso; Haidt, 2003) inducida experimentalmente tiene la capacidad de incrementar la motivación por ayudar a terceras personas (Schnall, Roper y Fessler, 2010). Los resultados de estos estudios son, 
por tanto, consistentes con la caracterización de la emoción prototípica como una respuesta afectiva que, no obstante, involucra ciertos componentes dimensionales característicos que le otorgan su particular estatus cualitativo.

\section{Discusión y conclusiones}

En el presente artículo, se ha realizado una revisión crítica de los estudios más relevantes en cognición y emoción que sostienen la viabilidad del modelo dimensional de Russell (2003). En particular, el análisis de cuatro hipótesis derivadas del modelo, sugiere que -en líneas generales- la propuesta de Russell constituye una alternativa que puede dar buena cuenta de una serie de cuestiones relevantes para el estudio de la emoción.

En primer lugar, la revisión de los estudios cognitivos y neuropsicológicos más recientes sugieren consistentemente una primacía del afecto básico (frente a los componentes más cognitivos) en el desarrollo temporal del episodio emocional (Hipótesis 1). En efecto, los estudios revisados reivindican la propuesta de Zajonc $(1980 ; 2000)$ según la cual las dimensiones básicas de la emoción preceden temporalmente a las evaluaciones cognitivas más sofisticadas (appraisal). De especial relevancia resultan los estudios más recientes que apuntan a la primacía temporal de la respuesta de arousal en lo que respecta al procesamiento del estímulo emocional (Rohr, Degner, y Wentura, 2014). La investigación enmarcada en el paradigma del priming afectivo (Almeida et al., 2013) y los estudios procedentes de la neurociencia (Wilson-Mendenhall et al., 2013) han corroborada la primera hipótesis derivada de la propuesta de Russell (2003), según la cual la respuesta de core affect constituye el primer paso de todo episodio emocional.

En segundo lugar, se analizó la evidencia empírica relacionada a la segunda hipótesis derivada del modelo de Russell, esto es, la presunta accesibilidad cognitiva del material hedónicamente congruente con el core affect experimentado. Décadas de investigación en priming afectivo han aportado información concluyente en este aspecto (Wong y Root, 2003). Si bien se debe señalar que existen diferentes posturas en lo que respecta al mecanismo preciso mediante el cual se produce el tan documentado "efecto priming" (Blaison et al., 2012.), los resultados obtenidos a través de diversos estudios soportan la hipótesis.

La investigación llevada a cabo a partir del Affective Misattribution Procedure (Payne et al., 2005) ha resultado de especial relevancia para el presente análisis, evidenciando que el atribuir erróneamente el core affect al estímulo emocional percibido es un fenómeno frecuente a nivel cognitivo (Hipótesis 3). El creciente interés académico en los diferentes estilos de pensamiento y sesgos cognitivos (Kahneman, 2011) se ha visto reflejado en la proliferación de estudios enmarcados en la automaticidad de la cognición social, y, en particular, en la influencia de los afectos incidentales en las respuestas cognitivas de tipo más complejo (juicios y toma de decisiones). Resulta de especial interés los estudios llevados a cabo en el marco de la cognición moral, por la diversidad de las metodologías empleadas y la naturaleza de sus resultados.

Para finalizar, la línea de investigación en el Appraisaltendency framework ha revelado la capacidad de las dimensiones más sofisticadas (o "cognitivas") implicadas en el episodio emocional para influir en diferentes dominios cognitivos, evidenciando de este modo la importancia de los componentes cognitivos en el episodio emocional (Oveis, Horberg, y Keltner, 2010). Ciertamente, los estudios desarrollados a partir de esta propuesta han resultado de especial utilidad para la caracterización del episodio emocional. Como consecuencia, la visión dimensional que reduce la experiencia de cada respuesta emocional a la intersección de coordenadas especificas en un espacio bidimensional (placer-displacer y activación-relajación) parece incapaz de dar cuenta de la complejidad inherente a la emoción (para revisiones ver Hamann, 2012; Reisenzein, 1994).

Por consiguiente, la presente revisión crítica encuentra viable la propuesta de un modelo dimensional en su versión integradora, según el cual la combinación de componentes dimensionales inespecíficos, tanto de tipo puramente afectivo (core affect) como de carácter más "cognitivo" (percepción de la cualidad afectiva, atribución, appraisal) constituyen ocasionalmente los prototipos emocionales, de la misma forma que la disposición de las estrellas conforman una gestalt que entendemos en términos de constelaciones.

Para finalizar, vale señalar que el análisis del modelo de Russell (2003) puede verse progresivamente revisado conforme se genere nueva evidencia empírica relevante a la problemática. Por ejemplo, aquellos estudios que aborden las diferencias culturales en el procesamiento emocional desde una perspectiva dimensional pueden resultar de utilidad para complementar el estudio de la relación entre core affect y meta-experiencia emocional. Si bien algunos estudios han abordado los efectos de la cultura en el procesamiento emocional básico (ChentsovaDutton y Tsai, 2010; Grossmann, Ellsworth, y Hong, 2012), consideramos que futuros estudios deberían profundizar en esta cuestión, por ejemplo, examinando la interacción entre diferentes contextos culturales y su posible influencia en la experimentación de los diferentes componentes dimensionales.

Adicionalmente, aquellos estudios que tengan como objeto el curso temporal de la respuesta empática (en cuanto se trata de un constructo que involucra componentes cognitivos y afectivos) pueden resultar de especial relevancia para mejorar el entendimiento del proceso de construcción psicológica de las respuestas emocionales.

\section{Referencias}

1. Adolphs, R. (2003). Cognitive neuroscience of human social behavior. Nature Reviews Neuroscience, 4, 165-178. http://dx.doi.org/10.1038/nrn1056

2. Almeida, J., Pajtas, P. E., Mahon, B. Z., Nakayama, K. \& 
Caramazza, A. (2013). Affect of the unconscious: Visually suppressed angry faces modulate our decisions. Cognitive, Affective, \& Behavioral Neuroscience, 13, 94-101. http:// dx.doi.org/10.3758/s13415-012-0133-7

3. Atkinson, A.P. \& Adolphs, R. (2005). Nonconscious emotions. Visual emotion perception. Mechanisms and processes. En L.F. Barrett, P.M. Niedenthal \& P. Winkielman (Comp.), Emotion and consciousness (pp. 150-182). New York: Guilford Press.

4. Bach, D. R., Hurlemann, R. \& Dolan, R. J. (2015). Impaired threat prioritization after selective bilateral amygdala lesions. Cortex, 63, 206-213. http://dx.doi.org/10.1016/j. cortex.2014.08.017

5. Barrett, L. F. (2012). Emotions are real. Emotion, 12, $413-$ 429. http://dx.doi.org/10.1037/a0027555

6. Beer, J. S., John, O., Scabini, D. \& Knight, R. T. (2006). Orbitofrontal cortex and social behavior. Integrating selfmonitoring and emotion cognition interactions. Journal of Cognitive Neuroscience, 18, 871-879. http://dx.doi. org/10.1162/jocn.2006.18.6.871

7. Blaison, C., Imhoff, R., Hühnel, I., Hess, U. \& Banse, R. (2012). The affect misattribution procedure: Hot or not? Emotion, 12, 403-412. http://dx.doi.org/10.1037/a0026907

8. Bower, G.H. (1981). Mood and memory. American Psychologist, 36, 129-148. http://dx.doi.org/10.1037/0003066X.36.2.129

9. Calder, A. J., Lawrence, A. D. \& Young, A. W. (2001). The Neuropsychology of Fear and Loathing. Nature Reviews Neuroscience, 2, 352-363. http://dx.doi. org/10.1038/35072584

10. Calder, A. J., Keane, J., Young, A. W., Lawrence, A. D., Mason, S. \& Barker, R. A. (2010). The relation between anger and different forms of disgust. Implications for emotion recognition impairments in Huntington's disease. Neuropsychologia, 48, 2719-2729. http://dx.doi.org/10.1016/j. neuropsychologia.2010.05.019

11. Chentsova-Dutton, Y. E. \& Tsai, J. L. (2010). Self-focused attention and emotional reactivity: The role of culture. Journal of Personality and Social Psychology, 98, 507-519. http://dx.doi.org/10.1037/a0018534

12. Clark, M. S. \& Isen, A. M. (1982). Toward understanding the relationship between feeling states and social behavior. Cognitive Social Psychology, 73, 108.

13. Clark, M. S. \& Isen, A. M. (1982). Toward understanding the relationship between feeling states and social behavior. En A. H. Hastrof \& A. M. Isen (Eds.), Cognitive social psychology (pp. 73-108). Amsterdam: Elsevier/ North-Holland.

14. Damasio, A. R. (1999). The feeling of what happens: Body and emotion in the making of consciousness. Harcourt Brace: Random House.

15. Darwin, C. (1872). The expression of emotions in animals and man. London: Murray. http://dx.doi. org/10.1037/10001-000
16. Diéguez-Risco, T., Aguado, L., Albert, J. \& Hinojosa, J. A. (2013). Faces in context: Modulation of expression processing by situational information. Social neuroscience, 8 , 601-620. http://dx.doi.org/10.1080/17470919.2013.834842

17. Dolan, R. J. (2002). Emotion, cognition, and behavior. Science, 298, 1191-1194. http://dx.doi.org/10.1126/science. 1076358

18. Doyen, S., Klein, O., Simons, D., Cleeremans, A. \& Cleeremans, A. (2014). On the other side of the mirror. Priming in cognitive and social psychology. Social Cognition, 32, 12-32. http://dx.doi.org/10.1521/soco.2014.32.supp.12

19. Ekman, P. (1999). Basic emotions. En T. Dalgleish y M. Power (Comp.) Handbook of cognition and emotion. Sussex: Wiley.

20. Ekman, P. \& Cordaro, D. T. (2011). What is meant by calling emotions basic. Emotion Review 3, 364-70. http:// dx.doi.org/10.1177/1754073911410740

21. Ellsworth, P. C. \& Scherer, K. R. (2003). Appraisal processes in emotion. En R. J. Davidson, K. R. Scherer \& H. Goldsmith (comps.), Handbook of Affective Sciences (pp. 572-95). Oxford: University Press.

22. Eskine, K. J., Kacinik, N. A. \& Prinz, J. J. (2011). A Bad Taste in the Mouth: Gustatory Disgust Influences Moral Judgments. Psychological Science, 22, 295-99. http:// dx.doi.org/10.1177/0956797611398497

23. Flexas, A., Rosselló, J., Christensen, J.F., Nadal, M., Olivera La Rosa, A. \& Munar, E. (2013). Affective Priming Using Facial Expressions Modulates Liking for Abstract Art. PLoS ONE 8, e80154. http://dx.doi.org/10.1371/journal.pone.0080154

24. Fukui, H. \& Toyoshima, K. (2014). Music increase altruism through regulating the secretion of steroid hormones and peptides. Medical hypotheses, 83, 706-708. http://dx.doi. org/10.1016/j.mehy.2014.09.023

25. Gawronski, B. \& Ye, Y. (2014). What drives priming effects in the affect misattribution procedure? Personality and Social Psychology Bulletin, 40, 3-15. http://dx.doi. org/10.1177/0146167213502548

26. Gendron, M., Roberson, D., van der Vyver, J. M. \& Barrett, L. F. (2014). Cultural relativity in perceiving emotion from vocalizations. Psychological science, 25, 911-920. http:// dx.doi.org/10.1177/0956797613517239

27. Gibbons, H. (2009). Evaluative priming from subliminal emotional words: insights from event-related potentials and individual differences related to anxiety. Consciousness and Cognition, 18, 383-400. http://dx.doi.org/10.1016/j. concog.2009.02.007

28. Grossmann, I., Ellsworth, P. C. \& Hong, Y. Y. (2012). Culture, attention, and emotion. Journal of Experimental Psychology: General, 141, 31-36. http://dx.doi.org/10.1037/ $\underline{\mathrm{a} 0023817}$

29. Gu, X., Liu, X., Van Dam, N. T., Hof, P. R. \& Fan, J. (2013). Cognition-emotion integration in the anterior insular cortex. Cerebral Cortex, 23, 20-27. http://dx.doi. 
org/10.1093/cercor/bhr367

30. Haidt, J. (2003). The moral emotions. In R. J. Davidson, K. R. Scherer \& H. H. Goldsmith (Comps.), Handbook of affective sciences (pp. 852-870). Oxford: Oxford University Press.

31. Hamann, S. (2012). Mapping discrete and dimensional emotions onto the brain: controversies and consensus. Trends in Cognitive Sciences, 16, 458-466. http:// dx.doi.org/10.1016/j.tics.2012.07.006

32. Han, S., Lerner, J. S. \& Keltner, D. (2007). Feelings and Consumer Decision Making: The Appraisal-Tendency Framework. Journal of Consumer Psychology, 17, 158168. http://dx.doi.org/10.1016/S1057-7408(07)70023-2

33. Hermans, D., Spruyt, A., De Houwer, J., \& Eelen, P. (2003). Affective priming with subliminally presented pictures. Canadian Journal of Experimental Psychology/Revue canadienne de psychologie expérimentale, 57, 97-114. http:// dx.doi.org/10.1037/h0087416

34. Higgins, E. T. (1998). The aboutness principle: A pervasive influence on human inference. Social Cognition, 16, 173198. http://dx.doi.org/10.1521/soco.1998.16.1.173

35. Izard, C. E. (2011). Forms and functions of emotions: Matters of emotion-cognition interactions. Emotion Review, 3, 371-78. http://dx.doi.org/10.1177/1754073911410737

36. James, W. (1884). What is an emotion? Mind, 9, 188-205. http://dx.doi.org/10.1093/mind/os-IX.34.188

37. Kahneman, D. (2011). Thinking, fast and slow. New York: Macmillan.

38. Keltner, D., Ellsworth P. C., \& Edwards, K. (1993). Beyond simple pessimism: Effects of sadness and anger on social perception. Journal of Personality \& Social Psychology, 64, 740-752. http://dx.doi.org/10.1037/0022-3514.64.5.740

39. Kirschner, S., \& Tomasello, M. (2010). Joint music making promotes prosocial behavior in 4-year-old children. Evolution and Human Behavior, 31, 354-364. http://dx.doi. org/10.1016/j.evolhumbehav.2010.04.004

40. Kokal I, Engel A, Kirschner S, Keysers C (2011). Synchronized Drumming Enhances Activity in the Caudate and Facilitates Prosocial Commitment - If the Rhythm Comes Easily. PLoS ONE 6, e27272. http://dx.doi.org/10.1371/ journal.pone.0027272

41. Kringelbach, M. L., Green, A. L., Owen, S. L., Schweder, P. M. \& Aziz, T. Z. (2010). Sing the mind electric-principles of deep brain stimulation. European Journal of Neuroscience, 32, 1070-1079. http://dx.doi.org/10.1111/j.14609568.2010.07419.x

42. Kuchinke, L., Krause, B., Fritsch, N. \& Briesemeister, B. B. (2014). A familiar font drives early emotional effects in word recognition. Brain and language, 137, 142-147. http://dx.doi.org/10.1016/j.bandl.2014.08.007

43. Kuppens, P., Champagne, D. \& Tuerlinckx, F. (2012). The dynamic interplay between appraisal and core affect in daily life. Frontiers in Psychology, 3, 1-8. http://dx.doi. org/10.3389/fpsyg.2012.00380
44. Lang, P. J. (1980). Behavioral treatment and bio-behavioral assessment: Computer applications. En J. B. Sidowski, J. H. Johnson, \& E. A Williams (Comps.), Technology in Mental Health Care Delivery Systems (pp. 119-137). Norwood, NJ: Ablex.

45. Lee, S. W. S. \& Schwarz, N. (2010). Dirty hands and dirty mouths: Embodiment of the moral-purity metaphor is specific to the motor modality involved in moral transgression. Psychological Science, 21, 1423-1425. http://dx.doi. org/10.1177/0956797610382788

46. Lerner, J. S., Goldberg, J. \& Tetlock, P. E. (1998). Sober second thought: The effects of accountability, anger, and authoritarianism on attributions of responsibility. Personality and Social Psychology Bulletin, 24, 563-574. http:// dx.doi.org/10.1177/0146167298246001

47. Lerner, J. S. \& Keltner, D. (2001). Fear, anger, and risk. Journal of Personality and Social Psychology, 81, 146-159. http://dx.doi.org/10.1037/0022-3514.81.1.146

48. Lin, D., Boyle, M. P., Dollar, P., Lee, H., Lein, E. S., Perona, P. \& Anderson, D. J. (2011). Functional identification of an aggression locus in the mouse hypothalamus. Nature, 470, 221-226. http://dx.doi.org/10.1038/nature09736

49. Lindquist, K. A., Wager, T. D., Kober, H., Bliss-Moreau, E. \& Barrett, L. F. (2012). The brain basis of emotion: a meta-analytic review. Behavioral and Brain Sciences, 35, 121-143. http://dx.doi.org/10.1017/S0140525X11000446

50. Lodge, M. \& Taber, C. S. (2005). The automaticity of affect for political leaders, groups, and issues: An experimental test of the hot cognition hypothesis. Political Psychology, 26, 455-482. http://dx.doi.org/10.1111/j.14679221.2005.00426.x

51. Meier, B. P., Schnall, S., Schwarz, N. \& Bargh, J. A. (2012). Embodiment in social psychology. Topics in cognitive science, 4, 705-716. http://dx.doi.org/10.1111/j.1756$\underline{8765.2012 .01212 . \mathrm{x}}$

52. G. Meilán, J., Carro, J., Guerrero, C., Carpi, A., Gómez, C. \& Palmero, F. (2012). El efecto de memoria congruente con el estado afectivo: reconocimiento diferencial de palabras de tristeza y alegría. Anales De Psicología, 28, 266-273.

53. Moons, W. G., Eisenberger, N. I. \& Taylor, S. E. (2010). Anger and fear responses to stress have different biological profiles. Brain, behavior, and immunity, 24, 215-219. http:// dx.doi.org/10.1016/j.bbi.2009.08.009

54. Motta, S. C., Goto, M., Gouveia, F. V., Baldo, M. V., Canteras, N. S. \& Swanson, L. W. (2009). Dissecting the brain's fear system reveals the hypothalamus is critical for responding in subordinate conspecific intruders. Proceedings of the National Academy of Sciences, 106, 4870-4875. http://dx.doi.org/10.1073/pnas.0900939106

55. Murphy, S. T. \& Zajonc, R. B. (1993). Affect, cognition, and awareness: Affective priming with optimal and suboptimal stimulus exposures. Journal of Personality and Social Psychology, 64, 723-739. http://dx.doi.org/10.1037/0022$\underline{3514.64 .5 .723}$ 
56. Niedenthal, P.M. (2008). Emotion concepts. En. M. Lewis, J.M. Haviland-Jones, \& L.F. Barrett (Comps.) Handbook of Emotions (pp. 587-600). New York, NY: Guilford Press.

57. Olivera La Rosa, A. \& Rosselló-Mir, J. (2012). Shocking moral judgments. Lap Lambert Saarbrücken: Academic Publishing.

58. Ousdal, O. T., Andreassen, O. A., Server, A. \& Jensen, J. (2014). Increased Amygdala and Visual Cortex Activity and Functional Connectivity towards stimulus novelty is associated with state anxiety. PloS one, 9, e96146. http:// dx.doi.org/10.1371/journal.pone.0096146

59. Oveis, C., Horberg, E. J. \& Keltner, D. (2010). Compassion, pride, and social intuitions of self-other similarity. Journal of Personality and Social Psychology, 98, 618-630. http://dx.doi.org/10.1037/a0017628

60. Paez, D. \& Carbonero, A. (1993). Afectividad, Cognición y Conducta social. Psicothema, 5, 133-150.

61. Palermo, R. \& Rhodes, G. (2007). Are you always on my mind? A review of how face perception and attention interact. Neuropsychologia, 45, 75-92. http://dx.doi. org/10.1016/j.neuropsychologia.2006.04.025

62. Paulmann, S. \& Pell, M. D. (2009). Decoding emotional faces depends on their representational value: ERP evidence. NeuroReport, 20, 1603-1608. http://dx.doi. org/10.1097/WNR.0b013e3283320e3f

63. Payne, B. K., Cheng, C. M., Govorun, O. \& Stewart, B. D. (2005). An inkblot for attitudes: Affect misattribution as implicit measurement. Journal of Personality and Social Psychology, 89, 277-293. http://dx.doi.org/10.1037/00223514.89.3.277

64. Phelps, E. A. (2006). Emotion and cognition: insights from studies of the human amygdala. Annual Review of Psychology, 57, 27-53. http://dx.doi.org/10.1146/annurev. psych.56.091103.070234

65. Prochnow, D., Kossack, H., Brunheim, S., Müller, K., Wittsack, H. J., Markowitsch, H. J. \& Seitz, R. J. (2013). Processing of subliminal facial expressions of emotion: A behavioral and fMRI study. Social neuroscience, 8, 448461. http://dx.doi.org/10.1080/17470919.2013.812536

66. Reisenzein, R. (1994). Pleasure-arousal theory and the intensity of emotions. Journal of Personality and Social Psychology, 67, 525-539. http://dx.doi.org/10.1037/00223514.67.3.525

67. Rohr, M., Degner, J. \& Wentura, D. (2012). Masked emotional priming beyond global valence activations. Cognition \& Emotion, 26, 224-244. http://dx.doi.org/10.1080/02 699931.2011 .576852

68. Rohr, M., Degner, J. \& Wentura, D. (2014). The "emotion misattribution" procedure: Processing beyond good and bad under masked and unmasked presentation conditions. Cognition \& Emotion, 29, 196-219. http://dx.doi.org/ $\underline{10.1080 / 02699931.2014 .898613}$

69. Rosselló, J. \& Revert, X. (2008). Modelos teóricos en el estudio científico de la emoción. En F. Palmero \& F. Martínez (Comps.) Motivación y emoción (pp. 95-137). Madrid: McGraw-Hill

70. Rovira, D. P. \& Martínez, A. J. C. (1993). Afectividad, cognición y conducta social. Psicothema, 5, 133-150.

71. Russell, J. A. (2003). Core affect and the psychological construction of emotion. Psychological Review, 110, 145172. http://dx.doi.org/10.1037/0033-295X.110.1.145

72. Russell, J. A. (2009). Emotion, core affect, and psychological construction. Cognition and Emotion, 23, 1259-1283. http://dx.doi.org/10.1080/02699930902809375

73. Russell, J. A. \& Barrett, L. F. (1999) Core affect, prototypical emotional episodes, and other things called emotion: Dissecting the elephant. Journal of Personality and Social Psychology, 76, 805-19. http://dx.doi.org/10.1037/00223514.76.5.805

74. Scarantino, A. (2012) Functional specialization does not require a one to-one mapping between brain regions and emotions. Behavioral and Brain Sciences, 35, 161-162. http://dx.doi.org/10.1017/S0140525X11001749

75. Schachter, S. \& Singer, J. (1962). Cognitive, social and physiological determinants of emotional state. Psychological Review, 69, 379-399. http://dx.doi.org/10.1037/ $\underline{\mathrm{h} 0046234}$

76. Scherer, K. R. (2001). The nature and study of appraisal: A review of the issues. En K.R. Scherar, A. Schorr \& T. Johnstone (Eds.), Appraisal Processes in emotion: Theory, methods, research (pp. 369-391). New York: Oxford University Press.

77. Schnall, S. (2011). Clean, proper and tidy are more than the absence of dirty, disgusting and wrong. Emotion review, 3, 264-266. http://dx.doi.org/10.1177/1754073911402397

78. Schnall, S., Benton, J. \& Harvey, S. (2008). With a clean conscience: Cleanliness reduces the severity of moral judgments. Psychological Science, 19, 1219-1222. http://dx.doi. org/10.1111/j.1467-9280.2008.02227.x

79. Schnall, S., Roper, J. \& Fessler, D. M. (2010). Elevation leads to altruistic behavior. Psychological science, 21, 315320. http://dx.doi.org/10.1177/0956797609359882

80. Schwarz, N. (2011). Feelings-as-information theory. En P.A.M. Van Lange, A.W. Kruglanski \& E.T. Higins (Eds.), Handbook of theories of social psychology (pp. 289-308). California: Sage.

81. Schimmack, U. \& Derryberry, D. E. (2005). Attentional interference effects of emotional pictures: threat, negativity, or arousal? Emotion, 5, 55-66. http://dx.doi. org/10.1037/1528-3542.5.1.55

82. Seidel, A. \& Prinz, J. (2013a). Mad and glad: Musically induced emotions have divergent impact on morals. Motivation and Emotion, 37, 629-637. http://dx.doi.org/10.1007/ $\underline{\text { s11031-012-9320-7 }}$

83. Seidel, A. \& Prinz, J. (2013b). Sound morality: Irritating and icky noises amplify judgments in divergent moral 
domains. Cognition, 127, 1-5. http://dx.doi.org/10.1016/j. cognition.2012.11.004

84. Shannon, M. L. \& Stark, C. P. (2003). The influence of physical appearance on personnel selection. Social Behavior and Personality: An International Journal, 31, 613-623. http://dx.doi.org/10.2224/sbp.2003.31.6.613

85. Smith, C. A. \& Ellsworth, P. C. (1985). Patterns of cognitive appraisal in emotion. Journal of Personality and Social Psychology, 48, 813-838. http://dx.doi.org/10.1037/00223514.48.4.813

86. Tsuchiya, N., Moradi, F., Felsen, C., Yamazaki, M. \& Adolphs, R. (2009). Intact rapid detection of fearful faces in the absence of the amygdala. Nature neuroscience, 12, 1224. http://dx.doi.org/10.1038/nn.2380

87. Vytal, K. \& Hamann, S. (2010). Neuroimaging support for discrete neural correlates of basic emotions: a voxel-based meta-analysis. Journal of Cognitive Neuroscience, 22, 2864-2885. http://dx.doi.org/10.1162/jocn.2009.21366

88. Wang, J., Xia, T., Xu, L., Ru, T., Mo, C., Wang, T. T. \& Mo, L. (2015). What is beautiful brings out what is good in you: The effect of facial attractiveness on individuals' honesty. International Journal of Psychology. (Publicado online: 17 Sep). http://dx.doi.org/10.1002/ijop.12218

89. Widen, S. C., Christy, A. M., Hewett, K. \& Russell, J. A. (2011). Do proposed facial expressions of contempt, shame, embarrassment, and compassion communicate the predicted emotion? Cognition \& Emotion, 25, 898-906. http://dx.doi.org/10.1080/02699931.2010.508270

90. Wilson-Mendenhall, C. D., Barrett, L. F. \& Barsalou, L. W. (2013). Neural Evidence That Human Emotions Share Core Affective Properties. Psychological Science, 24, 947-956. http://dx.doi.org/10.1177/0956797612464242

91. Winkielman, P., Berridge, K. C. \& Wilbarger, J. L. (2005). Unconscious affective reactions to masked happy versus angry faces influence consumption behavior and judgments of value. Personality and Social Psychology Bulletin, 1, 121-135. http://dx.doi.org/10.1177/0146167204271309

92. Wong, P. S. \& Root, J. C. (2003). Dynamic variations in affective priming. Consciousness and Cognition, 12, 147168. http://dx.doi.org/10.1016/S1053-8100(03)00007-2

93. Yang, Z. \& Tong, E. M. W. (2010). The effects of subliminal anger and sadness primes on agency appraisals. Emotion, 10, 915-922. http://dx.doi.org/10.1037/a0020306

94. Yang, J., Xu, X., Du, X., Shi, C. \& Fang, F. (2011). Effects of unconscious processing on implicit memory for fearful faces. PloS one, 6, e14641. http://dx.doi.org/10.1371/journal.pone.0014641

95. Zajonc, R. B. (1980). Feeling and thinking: preferences need no inferences. American Psychologist, 35, 151-175. http://dx.doi.org/10.1037/0003-066X.35.2.151

96. Zajonc, R. B. (2000). Feeling ad thinking: Closing the debate over the independence of affect. En J. P. Forgas (Comp.), Feeling and thinking: The role of affect in social cognition (pp. 31-58). Cambridge: Cambridge University Press.

Fecha de recepción: 29 de noviembre, 2015 Fecha de recepción de la versión modificada: 20 de febrero, 2016 Fecha de aceptación: 15 de marzo, 2016 